\title{
PERFIL DEL ALUMNADO DEL GRADO DE EDUCACIÓN INFANTIL: PROYECTO DOCENTE DE LA ASIGNATURA TRASTORNOS DEL DESARROLLO
}

\author{
M. Tamara Polo Sánchez \\ tpolo@ugr.es \\ Susana Tallón Rosarles \\ Miriam Hervás Torres \\ María Fernández Cabezas \\ Carolina Fernández Jiménez \\ Universidad de Granada
}

Fecha de Recepción: 2 Abril 2018

Fecha de Admisión: 10 Abril 2018

\section{RESUMEN}

Un aspecto relevante del contexto en el que se encuadra un proyecto docente es el relativo a las características específicas de las personas que van a protagonizar el proceso de aprendizaje: los alumnos de la titulación. Sin duda alguna, los alumnos son la materia prima de las universidades, y la razón de su existencia. Analizar las características de los alumnos, su procedencia, objetivos, intereses, motivaciones, aspiraciones personales, etc., son elementos imprescindibles en todo proyecto. En este trabajo se realiza un estudio del perfil de los alumnos universitarios de Educación Infantil en general, y de los alumnos de una materia objeto de estudio en particular, Trastornos del desarrollo. Su procedencia, expectativas e intereses formativos y profesionales, sus conocimientos previos y estilos de aprendizaje, condicionan las decisiones docentes. Las conclusiones extraídas de este análisis deberán verse reflejadas en las decisiones pedagógicas y metodológicas del plan docente.

Palabras clave: alumnado; educación infantil; perfil; planificación docente

\section{ABSTRACT}

Profile of the student of the children's degree of education: course teaching project development disorders.

A relevant aspect of the context in which a teaching project is framed is that related to the specific characteristics of the people who will be the protagonists of the learning process: the students of the degree program. Without a doubt, the students are the raw material of the universities, and the reason for their existence. Analyzing the characteristics of the students, their 
origin, objectives, interests, motivations, personal aspirations, etc., are essential elements in any project. In this work, a study is made of the university students of Pre-school Education in general, and of the students of the subject matter of study in particular, Development Disorders. Their origin, expectations and training and professional interests, their previous knowledge and learning styles, condition the teaching decisions. The conclusions drawn from this analysis should be reflected in the pedagogical and methodological decisions of the teaching plan.

Keywords: early childhood education; profile; students; teacher planning

\section{INTRODUCCIÓN}

El Grado de Educación Infantil (EI) proporciona la formación mediante la adquisición de competencias para desarrollar tareas de planificación, desarrollo y evaluación de actividades educativas dirigidas a niños y niñas de 0 a 6 años. No se exige ninguna formación previa específica más allá de las establecidas en artículo 14.1 del Real Decreto 1393/2007, de 29 de octubre, por el que se establece la ordenación de las enseñanzas universitarias oficiales, pero es recomendable un perfil científico social; es decir, preferentemente, formación en ciencias sociales; además de conocimientos en humanidades, artes, ciencias en general y en el área de la salud.

Respecto a las características académicas que debe reunir el alumnado que quiera acceder al grado, se establece que no requiere de ninguna prueba complementaria a las establecidas legalmente de carácter nacional. El acceso a las enseñanzas oficiales de Grado requerirá estar en posesión del título de bachiller o equivalente, y la superación de la prueba a la que se refiere el artículo 42 de la Ley Orgánica 6/2001, de Universidades, modificada por la Ley Orgánica 4/2007, de 12 de abril. Esta normativa ha sido posteriormente corregida por el Real Decreto 1892/2008, de 14 de noviembre, por el que se regulan las condiciones para el acceso a las enseñanzas universitarias oficiales de grado, y los procedimientos de admisión a las universidades públicas españolas, cuyo artículo 3 amplía las mencionadas modalidades de acceso. En este sentido, podrán acceder quienes se encuentren en alguna de las siguientes situaciones:

Haber superado la Prueba de Acceso a la Universidad (Selectividad).

Tener finalizado el COU (curso anterior al 74/75).

Haber superado las Pruebas de Madurez del Curso Preuniversitario - Bachillerato planes anteriores a 1953.

Haber finalizado Ciclos Formativos (Animación de Actividades físicas y Deportivas, Animación Social, Animación Turística, Educación Infantil, Integración Social e Interpretación de Lengua de Signos).

Ser Titulados Universitarios.

Haber superado la Prueba de Mayores de 25 años.

Los estudiantes extranjeros que hayan superado la Prueba de Acceso a la Universidad.

Los estudiantes procedentes de Estados miembros de la Unión Europea, o de otros Estados con Ios que España haya suscrito acuerdos internacionales al respecto, que cumplan los requisitos exigidos en su respectivo país para el acceso a la universidad.

En el título de Grado de Maestro en El elaborado por la UGR (verificado por ANECA, 2010) se recoge que el perfil del alumnado que quiera optar a la titulación de Grado en El, debería ser el de una persona que presente las siguientes características:

Interés por la educación, la infancia y la docencia.

Actitudes de respeto, responsabilidad, tolerancia, empatía, apertura y colaboración.

Valoración de la diversidad y de la multiculturalidad.

Creatividad y espíritu emprendedor.

Capacidad para comprender los procesos educativos, psicológicos, sociales, políticos, cultura- 
les y científicos junto con la capacidad para reflexionar sobre el papel que puede representar en la transformación de esos procesos.

Capacidad de atención, percepción y análisis.

Capacidad para trabajar en equipo.

Poseer habilidades comunicativas y de mediación.

La investigación sobre el perfil de los estudiantes de Magisterio no es nueva, no obstante, escasean las publicaciones en el contexto universitario español centradas en estudiantes de nuevo ingreso en El (Fernández-Molina, González y Del Molino, 2011). Entre los estudios realizados con estudiantes de magisterio de diferentes especialidades destaca el de Latorre y Pérez (2005). Un estudio con el propósito de identificar y dar a conocer perfiles característicos de sujetos entre los futuros maestros de la UGR de acuerdo con las creencias que poseen sobre la formación práctica universitaria, antes y después de su inmersión en los escenarios de prácticum. En esta vía, Mesa y Mingorance (2006), llevaron a cabo un estudio longitudinal desde el curso 2002/2003 hasta el 2005/2006, en el que participaron 412 alumnos de nuevo ingreso en las distintas especialidades de la Diplomatura de Magisterio en la Facultad de Educación y Humanidades de Melilla. Con tal motivo se elaboró una encuesta de carácter anónimo con ítems del tipo estado civil, género, edad, lugar de residencia habitual, profesión del padre y de la madre, en la categoría de datos sociológicos; en cuanto a datos de tipo académico se preguntó sobre estudios previos realizados, opción de estudios durante el bachillerato, nota media de bachillerato y de selectividad, orden de prioridad en la elección de estudios de magisterio, momento en que se decidió a estudiar magisterio, motivos por los que se eligió esta carrera, y por último, expectativas de continuar un segundo ciclo universitario.

Bertomeu, Canet, Gil y Jarabo (2006) analizaron las distintas motivaciones que determinan la elección de las diferentes especialidades de la carrera de magisterio (Educación Física, Educación Primaria, Educación Infantil, Educación Musical y Educación Especial), y las posibles relaciones que puedan aparecer entre dichas especialidades. Para llevar a cabo la investigación, que se realizó durante el curso escolar 2006/2007, se tomaron como muestra los estudiantes de la Universitat Jaume I de Castellón y la Universitat de Valencia. Se trata de un trabajo de carácter descriptivo con el que se pretendían obtener valoraciones y propuestas útiles para la orientación y la formación de los futuros maestros. La hipótesis que se planteó fue que la elección de las distintas especialidades de magisterio dependería de las motivaciones de cada alumno, y éstas se agruparían en función de la elección de la especialidad.

Camina y Salvador (2007), analizaron las características y condicionamientos que mostraban los estudiantes de las especialidades de Educación Infantil, Primaria, Lengua Extranjera, Educación Musical y Educación Física impartidas en la Universidad Autónoma de Madrid (edad, estudios previos, modalidad de acceso a la universidad, motivaciones, intereses, gustos y aficiones por actividades relacionadas con profesiones, y rasgos más característicos de su autoimagen).

En la Facultad de Ciencias de la Educación de la UGR, López-Justicia, Hernández, Fernández, Polo y Chacón (2008), indagaron en el perfil de las características formativas y socio-afectivas de los alumnos de nuevo ingreso de las diferentes especialidades de Educación. Participaron un total de 203 estudiantes que fueron evaluados en vocabulario, comprensión lectora y ortografía, autoconcepto y habilidades sociales. También se recogieron datos sobre variables sociodemográficas. Y más reciente en el tiempo Fernández-Molina et al. (2011) realizaron un estudio con objeto de conocer el perfil y competencias iniciales de los estudiantes que acceden a la Universidad de Málaga por la titulación de El. De igual modo se ha indagado en perfiles específicos tales como el de maestro de primaria especialista en educación musical (Prieto, 2001) y educación física (Zurita et al., 2016). 


\section{OBJETIVO}

En el presente estudio, se pretende conocer específicamente el perfil de acceso y competencias iniciales del estudiante que cursa la materia de El de segundo curso, Trastornos del Desarrollo de la FCCE de la UGR.

\section{PARTICIPANTES}

Desde el curso 2012/2013 hasta el 2016/2017 se encuestó a 1277 mujeres (92,5\%), y 63 hombres (4,6\%). Un total de 1340 alumnos que informarían sobre su procedencia educativa, su experiencia y motivación por la Universidad, por la El como carrera y como profesión, y sus competencias (Tabla 1).

Tabla 1

Distribución de la muestra por curso. Perfil de los estudiantes de Educación Infantil

\begin{tabular}{|c|c|c|c|c|c|c|c|}
\hline & Grupo curso & $\mathbf{A}$ & B & $\mathbf{C}$ & D & $\mathbf{E}$ & Total \\
\hline \multirow{3}{*}{$2012 / 13$} & Muestra & 63 & 58 & 63 & 53 & 31 & 268 \\
\hline & $\%$ & 23,5 & 21,6 & 23,5 & 19,8 & 11,6 & 100 \\
\hline & Matriculados & 65 & 67 & 65 & 66 & 41 & 304 \\
\hline \multirow{3}{*}{$2013 / 14$} & Muestra & 65 & 63 & 68 & 45 & 44 & 285 \\
\hline & $\%$ & 22,8 & 22,1 & 23,9 & 15,8 & 15,4 & 100 \\
\hline & Matriculados & 66 & 68 & 69 & 67 & 58 & 328 \\
\hline \multirow{3}{*}{$2014 / 15$} & Muestra & 66 & 62 & 61 & 39 & 37 & 265 \\
\hline & $\%$ & 24,9 & 23,4 & 23 & 14,7 & 14 & 100 \\
\hline & Matriculados & 66 & 69 & 70 & 65 & 51 & 321 \\
\hline \multirow{3}{*}{$2015 / 16$} & Muestra & 60 & 55 & 64 & 33 & 41 & 253 \\
\hline & $\%$ & 23,7 & 21,7 & 25,3 & 13 & 16,2 & 100 \\
\hline & Matriculados & 69 & 70 & 70 & 69 & 54 & 332 \\
\hline \multirow{3}{*}{$2016 / 17$} & Muestra & 50 & 55 & 61 & 58 & 45 & 269 \\
\hline & $\%$ & 18,6 & 20,4 & 22,7 & 21,6 & 16,7 & 100 \\
\hline & Matriculados & 68 & 66 & 68 & 68 & 58 & 328 \\
\hline
\end{tabular}

\section{METOdOLOGíA}

\section{Instrumento}

Se llevó a cabo una metodología secuencial transversal, exploratoria y descriptiva, concretamente para la recogida de los datos se utilizó el cuestionario Perfil de Acceso del Alumnado Universitario de Educación Infantil de Fernández-Molina et al. (2011). Este cuestionario "ad hoc" consta de preguntas de opción cerrada (Sí-No; Ninguna-Alguna-Mucha) y de preguntas con varios niveles de respuesta para cada ítem (desde 0 , que significa "totalmente en desacuerdo, nada" a 5 que significa "totalmente de acuerdo, todo"). Las dimensiones y variables analizadas se muestran en la Tabla 2. 
Tabla 2

Dimensiones y Variables Analizadas (Fernández-Molina et al., 2011)

\begin{tabular}{|l|l|}
\hline \multicolumn{1}{|c|}{ Dimensiones } & \multicolumn{1}{c|}{ Variables } \\
\hline A. Procedencia & A.1 Nivel educativo previo no universitario \\
\hline B. Experiencia y & B.1 Estudios universitarios previos \\
motivación por la & B.2 Motivación para elegir el estudio universitario \\
Universidad & b.2.1 Prestigio social \\
& b.2.2 Formación cultural general \\
& b.2.3 Conocimiento de nuevas personas y ambientes \\
& b.2.4 Puesto de trabajo \\
& b.2.5 Desarrollo de capacidad intelectual \\
& b.2.6 Aportación a la sociedad \\
\hline C. Experiencia y & \\
motivación por la EI, & C.1 Motivación para elegir la carrera de EI \\
como carrera y como & c.1.2 Nota \\
profesión & c.1.3 Profesión \\
& c.1.4 Puente \\
& c.1.6 Carrera corta \\
& c.1.7 Interés \\
& c.1.8 Igualdad \\
& \\
\hline D. Ideas previas sobre la & D.1 EI como carrera \\
& d.1.1 Dificultad elevada \\
& d.1.2 Mucho tiempo de trabajo \\
& d.1.3 Sólo asistencia a clase para aprobar \\
& d.1.4 Metodología por trabajos en grupo y clases participativas \\
& d.1.5 Estudio memorístico de mucho temario \\
& d.1.6 Estudio razonado \\
\hline E. Competencias para el & \\
EI & E.1 Instrumentales de acceso \\
& e.1.1 Expresión escrita \\
& e.1.2 Competencia Lectora \\
& e.1.3 Vocabulario \\
& e.1.4 Expresión oral \\
& e.1.5 Técnicas de estudio \\
& e.1.6 Hábito lector \\
& E.2 Conocimiento y uso de TICS \\
& e.2.1 Manejo con el Programa Word \\
& e.2.2 Navegar Internet \\
& e.2.3 Seleccionar información \\
& e.2.4 Correo electrónico \\
& e.2.5 Foros virtuales \\
& \\
&
\end{tabular}

\section{RESULTADOS}

A continuación se comentan los resultados más destacados del estudio realizado.

Procedencia: alude al nivel educativo no universitario cursado con anterioridad a la carrera de El. La mayor parte del alumnado encuestado procedía de Bachillerato $(55,1 \%)$, y de Ciclos 
Formativos de Grado Superior (23,7\%), aunque hay un $14,8 \%$ que contaba con ambas titulaciones. Un $6 \%$ provenía de otras vías, por ejemplo, de la prueba de acceso a mayores de 25 años, y el menor porcentaje $(0,4 \%)$ lo representaban los estudiantes que accedían del antiguo BUP.

Tabla 3

Estudios no universitarios

\begin{tabular}{lcc}
\hline & Frecuencia & Porcentaje \\
\hline Bachiller & 738 & 55,1 \\
Ciclo Formativo de Grado Superior & 318 & 23,7 \\
BUP & 6 &, 4 \\
Otros & 80 & 6 \\
Bachiller y CFGS & 198 & 14,8 \\
Total & 1340 & 100 \\
\hline
\end{tabular}

Experiencia y motivación por la Universidad: se refiere el haber cursado estudios universitarios con anterioridad, habiéndolos finalizado o no, la carrera de El, y la influencia de diferentes tipos de motivación en la decisión de cursar una carrera universitaria. En líneas generales, los encuestados no habían cursado ni poseían estudios universitarios previos $(90,4 \%)$.

Tabla 4

Estudios universitarios previos

\begin{tabular}{lcc}
\hline & Frecuencia & Porcentaje \\
\hline $\mathrm{Si}$ & 129 & 9,6 \\
$\mathrm{No}$ & 1211 & 90,4 \\
Total & 1340 & 100 \\
\end{tabular}

Respecto a la principal motivación para los estudiantes de El cuando decidían cursar una carrera universitaria, era obtener un puesto de trabajo $(57,3 \%)$, seguido de la aspiración a aportar lo mejor de sí a la sociedad (27,1\%).

Tabla 5

Motivación para elegir Educación Infantil

\begin{tabular}{lcc}
\hline & Frecuencia & Porcentaje \\
\hline Me proporciona prestigio social & 8 &, 6 \\
Quiero recibir una formación cultural general & 125 & 9,1 \\
Deseo conocer nuevas personas, ambientes, etc. & 20 & 1,4 \\
Me gustaría formarme profesionalmente para & 791 & 57,3 \\
conseguir un puesto de trabajo & 22 & 1,6 \\
Necesito desarrollar mi capacidad intelectual & 374 & 27,1 \\
Aspiro a aportar lo mejor de mí a la sociedad & 1340 & 100 \\
Total & & \\
\hline
\end{tabular}


Experiencia y motivación por la El: alude a si se habían matriculado con anterioridad en esta titulación, si habían tenido experiencias, remuneradas económicamente o no, de educación y crianza de niños pequeños y si conocían la profesión. En motivación se ha considerado si habían elegido El como primera opción, y el grado en que distintos tipos de motivación les han influido al elegir El.

El $91,5 \%$ del alumnado participante puso de manifiesto que se matriculaba por primera vez en el grado, frente al 8,5\% que era su segunda matrícula. Además un alto porcentaje (89,9\%) afirmó haber elegido la carrera como primera opción, frente al 10,1\% restante. Asimismo, el $91 \%$ tuvo conocimiento de la profesión por familiares y amigos que trabajan o dirigen su propio centro de El, en cambio el $9 \%$ dijo no tener conocimientos del ejercicio de la El. Finalmente, el $53,3 \%$ y $15,3 \%$ señaló que ha tenido alguna o muchas experiencias como educador de niños pequeños, frente al $31,4 \%$.

Respecto a los motivos señalados para elegir la carrera de El frente a otras opciones, tal y como puede visualizarse en la Tabla 6 , destacan los siguientes resultados:

Tabla 6

Tipos de motivacion sobre la carrera de Educacion Infantil

\begin{tabular}{|c|c|c|c|c|c|c|c|c|c|c|c|c|c|c|}
\hline & \multicolumn{2}{|c|}{$\begin{array}{c}\text { Nota } \\
\text { insuficiente }\end{array}$} & \multicolumn{2}{|c|}{$\begin{array}{c}\text { Encanta la } \\
\text { profesión }\end{array}$} & \multicolumn{2}{|c|}{$\begin{array}{c}\text { Puente } \\
\text { para } \\
\text { acceder a } \\
\text { una carrera } \\
\text { superior }\end{array}$} & \multicolumn{2}{|c|}{$\begin{array}{l}\text { Aptitudes } \\
\text { para } \\
\text { enseñar }\end{array}$} & \multicolumn{2}{|c|}{$\begin{array}{l}\text { Carrera corta } \\
\text { y asequible }\end{array}$} & \multicolumn{2}{|c|}{$\begin{array}{l}\text { Interés por } \\
\text { temas de la } \\
\text { infancia }\end{array}$} & \multicolumn{2}{|c|}{$\begin{array}{l}\text { Conseguir } \\
\text { igualdad } \\
\text { social y } \\
\text { educativa }\end{array}$} \\
\hline & $\mathrm{F}$ & $\%$ & $\mathrm{~F}$ & $\%$ & $\mathrm{~F}$ & $\%$ & $\mathrm{~F}$ & $\%$ & $\mathrm{~F}$ & $\%$ & $\mathrm{~F}$ & $\%$ & $\mathrm{~F}$ & $\%$ \\
\hline $\mathrm{NC}$ & 157 & 11,7 & 38 & 2,8 & 81 & 6 & 46 & 3,4 & 76 & 5,7 & 43 & 3,5 & 49 & 3,7 \\
\hline TD & $\begin{array}{l}105 \\
3\end{array}$ & 78,6 & 60 & 4,5 & 857 & 64 & 52 & 3,9 & 971 & 72,5 & 47 & 3,5 & 14 & 1 \\
\hline ED & 46 & 3,4 & 9 & 0,7 & 177 & 13,2 & 26 & 1,9 & 128 & 9,6 & 31 & 2,3 & 44 & 3,3 \\
\hline NA & 34 & 2,5 & 46 & 3,4 & 119 & 8,9 & 86 & 6,4 & 89 & 6,6 & 91 & 6,8 & 154 & 11,5 \\
\hline DA & 22 & 1,6 & 164 & 12,2 & 35 & 2,6 & 449 & 33,5 & 29 & 2,2 & 221 & 16,5 & 323 & 24,1 \\
\hline TA & 28 & 2 & 1023 & 76,3 & 71 & 5,3 & 681 & 50,8 & 47 & 3,5 & 907 & 67,7 & 756 & 56,4 \\
\hline
\end{tabular}

El no haber tenido nota suficiente para cursar otra carrera $(78,6 \%)$, el hecho de ser una carrera corta y de coste económico asequible $(72,5 \%)$, o que sirvan de puente para acceder a una carrera superior (64\%), son motivos que no les influyeron. En cambio, el gusto por la profesión de educar $(76,3 \%)$, el interés por los temas de la infancia $(67,7 \%)$, querer ayudar a conseguir más igualdad social y educativa en la infancia $(56,4 \%)$, o creer tener aptitudes para enseñar a los niños pequeños $(50,8 \%)$ son los motivos que más les influyeron.

Ideas previas sobre la El: contempla de un lado las ideas implícitas que tenían sobre la El como carrera, fundamentalmente relacionadas con las exigencias para aprobar las asignaturas y con el tipo de metodología docente, de otro las ideas previas sobre la El pero como profesión, donde se les pregunta sobre el concepto que tienen del oficio y sobre las exigencias que creen que tiene el ejercicio de la El.

En la Tabla 7 se plasman los resultados encontrados respecto a las ideas previas sobre la titulación de los alumnos de la asignatura de TD. La mayor parte de los mismos, consideraron que el nivel de dificultad de la carrera de El no era ni poco ni excesivamente elevado $(39,3 \%)$, en cambio 
consideraron que hay que dedicarle mucho tiempo de trabajo $(32,9 \%)$, por lo que no basta con asistir a clase para aprobar $(66,6 \%)$. Se mostraron generalmente neutrales respecto a la cuestión de si es necesario memorizar mucho temario para el examen $(30,4 \%)$, o que requieran un alto nivel de estudio razonado $(35,4 \%)$.

Tabla 7

Ideas previas sobre la carrera de Educación Infantil

\begin{tabular}{|c|c|c|c|c|c|c|c|c|c|c|c|c|}
\hline & \multicolumn{2}{|c|}{$\begin{array}{c}\text { Dificultad } \\
\text { elevada }\end{array}$} & \multicolumn{2}{|c|}{$\begin{array}{c}\text { Dedicarle } \\
\text { mucho } \\
\text { tiempo }\end{array}$} & \multicolumn{2}{|c|}{$\begin{array}{l}\text { Para aprobar } \\
\text { solo es } \\
\text { necesario ir } \\
\text { a clase }\end{array}$} & \multicolumn{2}{|c|}{$\begin{array}{c}\text { Trabajos en } \\
\text { grupo y } \\
\text { clases } \\
\text { participativa } \\
\text { s }\end{array}$} & \multicolumn{2}{|c|}{$\begin{array}{l}\text { Memorizar } \\
\text { temario y } \\
\text { soltarlo en } \\
\text { el examen }\end{array}$} & \multicolumn{2}{|c|}{$\begin{array}{l}\text { Alto nivel } \\
\text { de estudio } \\
\text { razonado }\end{array}$} \\
\hline & $\mathrm{F}$ & $\%$ & $\mathrm{~F}$ & $\%$ & $\mathrm{~F}$ & $\%$ & $\mathrm{~F}$ & $\%$ & $\mathrm{~F}$ & $\%$ & $\mathrm{~F}$ & $\%$ \\
\hline $\mathrm{NC}$ & 61 & 4,6 & 35 & 2,6 & 57 & 4,3 & 34 & 2,5 & 45 & 3,4 & 41 & 3,1 \\
\hline TD & 301 & 22,5 & 59 & 4,4 & 893 & 66,6 & 68 & 5,1 & 323 & 24,1 & 94 & 7 \\
\hline ED & 302 & 22,5 & 78 & 5,8 & 197 & 14,7 & 62 & 4,6 & 393 & 29,3 & 202 & 15,1 \\
\hline NA & 527 & 39,3 & 303 & 22,6 & 102 & 7,6 & 178 & 13,3 & 407 & 30,4 & 474 & 35,4 \\
\hline DA & 101 & 7,5 & 424 & 31,6 & 60 & 4,5 & 399 & 29,8 & 109 & 8,1 & 318 & 23,7 \\
\hline TA & 48 & 3,6 & 441 & 32,9 & 31 & 2,3 & 599 & 44,7 & 63 & 4,7 & 211 & 15,7 \\
\hline
\end{tabular}

El 64,5\% expresó su desacuerdo respecto al hecho de que sea una profesión donde se gane suficiente y se trabaje poco. Así, opinaron que es un trabajo de mucha importancia social $(49,2 \%)$, que requiere de paciencia y vocación $(78,8 \%)$, así como de actualización permanente $(61,5 \%)$ (Tabla 8).

Tabla 8

Ideas previas sobre la profesión de Educación Infantil

\begin{tabular}{ccccccccccccc}
\hline & $\begin{array}{c}\text { Se gana } \\
\text { suficiente y se } \\
\text { trabaja poco }\end{array}$ & $\begin{array}{c}\text { Trabajo que } \\
\text { tiene mucha } \\
\text { importancia } \\
\text { social }\end{array}$ & $\begin{array}{c}\text { Oficio } \\
\text { cómodo y } \\
\text { seguro }\end{array}$ & $\begin{array}{c}\text { Formar a los } \\
\text { niños para } \\
\text { el futuro }\end{array}$ & $\begin{array}{c}\text { Paciencia y } \\
\text { vocación }\end{array}$ & $\begin{array}{c}\text { Actualización } \\
\text { permanente }\end{array}$ \\
\hline NC & 79 & 5,9 & 49 & 3,7 & 42 & 3,1 & 32 & 2,4 & 33 & 2,5 & 40 & 3 \\
TD & 864 & 64,5 & 111 & 8,3 & 276 & 20,6 & 20 & 1,5 & 4 & 0,3 & 11 & 0,8 \\
ED & 210 & 15,7 & 113 & 8,4 & 286 & 21,3 & 18 & 1,3 & 10 & 0,7 & 36 & 2,7 \\
NA & 133 & 9,9 & 161 & 12 & 415 & 31 & 44 & 3,3 & 51 & 3,8 & 130 & 9,7 \\
DA & 34 & 2,5 & 247 & 18,4 & 206 & 15,4 & 172 & 12,8 & 186 & 13,9 & 299 & 22,3 \\
& 20 & 1,5 & 659 & 49,2 & 115 & 8,6 & 1054 & 78,7 & 1056 & 78,8 & 824 & 61,5 \\
\hline
\end{tabular}

Competencias para el estudio de la carrera de El: se toman en cuenta tanto las competencias intelectuales básicas de acceso a estudios superiores (hábito lector, expresión escrita y oral, 
comprensión lectora, hábitos de estudio, etc.), y las tecnologías de la información y la comunicación más utilizadas en las metodologías de enseñanza-aprendizaje en la universidad, incluyéndose el conocimiento y uso de las mismas.

Cuando se les pregunta por las competencias instrumentales de acceso para el estudio de El, consideraron que se expresaban bien por escrito, a nivel ortográfico y gramatical $(41,4 \%)$, poseen un vocabulario óptimo (47,6\%), comprenden las ideas fundamentales de un texto escrito $(50,7 \%)$, para lo cual hacen uso de técnicas de estudio $(45,6 \%)$.

Tabla 9

Competencias Instrumentales de Acceso para el estudio de la carrera de Educación Infantil

\begin{tabular}{ccccccccccccc}
\hline & $\begin{array}{c}\text { Expresión } \\
\text { Escrita }\end{array}$ & \multicolumn{2}{c}{$\begin{array}{c}\text { Competencia } \\
\text { lectora }\end{array}$} & Vocabulario & $\begin{array}{c}\text { Expresión } \\
\text { oral }\end{array}$ & $\begin{array}{c}\text { Técnicas } \\
\text { de estudio }\end{array}$ & Hábito lector \\
& F & $\%$ & F & $\%$ & F & $\%$ & F & $\%$ & F & $\%$ & F & $\%$ \\
\hline NC & 39 & 2,9 & 36 & 2,7 & 37 & 2,8 & 36 & 2,7 & 33 & 2,5 & 51 & 3,8 \\
TD & 18 & 1,3 & 11 & 0,8 & 26 & 1,9 & 24 & 1,8 & 39 & 2,9 & 145 & 10,8 \\
ED & 67 & 5 & 39 & 2,9 & 54 & 4 & 87 & 6,5 & 57 & 4,3 & 255 & 19 \\
NA & 324 & 23,5 & 243 & 18,1 & 419 & 31,3 & 423 & 31,6 & 239 & 17,8 & 426 & 31,8 \\
DA & 572 & 41,4 & 680 & 50,7 & 638 & 47,6 & 597 & 44,6 & 361 & 26,9 & 239 & 17,8 \\
TA & 320 & 23,2 & 331 & 24,7 & 166 & 12,4 & 173 & 12,9 & 611 & 45,6 & 224 & 16,7 \\
\end{tabular}

En la Tabla 10, se recogen las competencias relativas al conocimiento y uso de las TICs, así la mayor parte de los estudiantes encuestados, señalaron conocer y hacer uso de Word $(60,6 \%)$, Internet $(74,2 \%)$, correo electrónico $(68,7 \%)$, y de los foros virtuales $(28,4 \%)$, frente a la construcción de páginas webs $(42,4 \%)$ o uso de las tutorías virtuales $(39,3 \%)$, que parecen conocer en menor medida.

Tabla 10

Competencias Conocimiento y Uso de las Tics para el Estudio de la Carrera de Educación Infantil. Distribución de sujetos por niveles de respuesta. Frecuencia y Porcentaje

\begin{tabular}{ccccccccccccccc}
\hline & $\begin{array}{c}\text { Programa } \\
\text { Word }\end{array}$ & \multicolumn{2}{c}{$\begin{array}{c}\text { Navegar } \\
\text { Internet }\end{array}$} & $\begin{array}{c}\text { Seleccionar } \\
\text { información }\end{array}$ & $\begin{array}{c}\text { Correo } \\
\text { electrónico }\end{array}$ & $\begin{array}{c}\text { Foros } \\
\text { virtuales }\end{array}$ & $\begin{array}{c}\text { Construcción } \\
\text { de webs }\end{array}$ & $\begin{array}{c}\text { Tutorías } \\
\text { virtuales }\end{array}$ \\
\hline & F & $\%$ & F & $\%$ & F & $\%$ & F & $\%$ & F & $\%$ & F & $\%$ & F & $\%$ \\
\hline NC & 36 & 2,7 & 27 & 2 & 29 & 2,1 & 40 & 3 & 53 & 4 & 70 & 5,2 & 71 & 5,3 \\
TD & 13 & 1 & 8 & 0,6 & 11 & 0,8 & 7 & 0,5 & 183 & 13,7 & 568 & 42,4 & 526 & 39,3 \\
ED & 28 & 2,1 & 11 & 0,8 & 28 & 2 & 21 & 1,6 & 198 & 14,8 & 286 & 21,3 & 317 & 23,7 \\
NA & 122 & 9,1 & 57 & 4,1 & 192 & 13,9 & 82 & 6,1 & 304 & 22,7 & 241 & 18 & 226 & 16,9 \\
DA & 328 & 24,5 & 213 & 15,4 & 541 & 39,2 & 270 & 20,1 & 222 & 16,6 & 90 & 6,7 & 111 & 8,3 \\
TA & 812 & 60,6 & 1024 & 74,2 & 539 & 39,1 & 920 & 68,7 & 380 & 28,4 & 85 & 6,3 & 89 & 6,6 \\
\hline
\end{tabular}




\section{PERFIL DEL ALUMNADO DEL GRADO DE EDUCACIÓN INFANTIL: PROYECTO DOCENTE DE LA ASIGNATURA TRASTORNOS DEL DESARROLLO}

\section{DISCUSIÓN Y CONCLUSIONES}

La elección por cursar estudios universitarios de El puede estar condicionada por razones muy diversas tales como la personalidad del sujeto u elementos del entorno (influencia familiar o de otros grupos, modelos de referencia, investigaciones sociales, tendencias sociales, etc.). En los últimos años los grados de magisterio están siendo los más demandados (Bertomeu, et al., 2006; García, 2012), por tal razón con este estudio, se pretenden describir las motivaciones y el perfil de los estudiantes universitarios que eligen el grado de El, lo que ayudará a la planificación didáctica del docente tomando como referente el perfil de dicho alumnado, concretamente el de Trastornos del desarrollo.

Nos encontramos en general, al igual que en un estudio similar realizado en la Universidad de Málaga (Fernández-Molina et al., 2011) ante un grupo mayoritariamente de alumnas que han cursado Bachillerato, y/o Ciclos Formativos de Grado Superior, que deciden cursar una carrera universitaria fundamentalmente para obtener un puesto de trabajo, que eligen El como primera opción, y no tienen experiencia previa en otras carreras.

Entre los motivos señalados para elegir la carrera de El, en consonancia con los datos aportados por Mesa y Mingorance (2006), destacan el gusto por la profesión de educar. Así, opinaron que es un trabajo de mucha importancia social que requiere de paciencia y vocación, así como actualización permanente. Además de que es una carrera a la que hay que dedicarle mucho tiempo de trabajo, por lo que no basta con asistir a clase para aprobar.

En esta vía, y respecto a las competencias instrumentales tales como el conocimiento y uso de las TICs, la mayor parte de los estudiantes encuestados de la asignatura Trastornos del Desarrollo, señalaron conocer y hacer uso de Word, Internet, correo electrónico o foros virtuales frente a la construcción de páginas webs o uso de las tutorías virtuales que parecen conocer en menor medida.

La investigación sobre el perfil de los estudiantes de Magisterio no es nueva, no obstante, escasean las publicaciones en el contexto universitario español centradas en estudiantes de nuevo ingreso en El (Fernández-Molina et al., 2011). Por lo que, se señala la necesidad de realizar nuevos estudios sobre la temática tratada, para de esta manera poder contrastar los resultados y aumentar el nivel de conocimiento sobre el tema.

Los resultados obtenidos en esta investigación quedarán de un lado, a disposición de profesionales de orientación, permitiéndoles conocer los diferentes perfiles de las personas que eligen las distintas especialidades de los estudios de magisterio y que basándose en ellos, puedan guiar y orientar académica, vocacional y profesionalmente a sus alumnos (Bertomeu, et al., 2006). Además, permitirán al docente del grado de El un mayor conocimiento de cara a su planificación didáctica.

\section{REFERENCIAS BIBLIOGRÁFICAS}

Agencia Nacional de Evaluación de la Calidad y Acreditación - ANECA (2004). Libro Blanco. Título

de Grado en Magisterio (Vol. 1). Madrid. Recuperado de http://www.aneca.es/ var/media/150404/libroblanco_jun05_magisterio1.pdf

Agencia Nacional de Evaluación de la Calidad y Acreditación - ANECA (2010). Grado en Maestro de Educación Infantil. Universidad de Granada. Madrid: Agencia Nacional de Evaluación de la Calidad y Acreditación. BOE (2007). ORDEN ECI/3854/2007, de 27 de diciembre, por la que se establecen los requisitos para la verificación de los títulos universitarios oficiales que habiliten para el ejercicio de la profesión de Maestro en Educación Infantil. Recuperado de http://grados.ugr.es/infantil/pages/infoacademica/42maestroeducacioninfantilverificado

Bertomeu, F.J., Canet, G., Gil, V. y Jarabo, J.A. (2006). Las motivaciones hacia los estudios de magisterio. Fòrum de recerca, 12, 1-16. Recuperado de http://repositori.uji.es/ 
xmlui/hitstream/handle/10234/78666/forum_2006_32.pdf?sequence=1

Camina, A. y Salvador, I. (2007). Condicionantes y características de los estudiantes que inician Magisterio. Estudio descriptivo y comparativo entre especialidades. Tendencias pedagógicas, 12, 245-262.

Fernández-Molina, M., González, V. y Del Molino, G. (2011). Perfil del alumnado universitario de Educación Infantil. Un estudio descriptivo desde los inicios del Espacio Europeo de Educación Superior hasta los Estudios de Grado (2006-2010). Revista de Investigación Educativa, 29 (1), 187-203.

García, A. (2012). Las carreras más demandadas y con más salidas. Recuperado de http://www.consumer.es/web/es/educacion/universidad/2012/09/14/213348.php

Latorre, M. J. y Pérez, P. (2005). El perfil del estudiante de Magisterio y su formación práctica universitaria. Revista Qurriculum, 18, 255-274.

Ley Orgánica 4/2007, de 12 de abril, por la que se modifica la Ley Orgánica 6/2001, de 21 de diciembre, de Universidades. Recuperado de https://www.boe.es/boe/dias/2007/04/13/pdfs/A1624116260.pdf

Ley Orgánica 6/2001, de 21 de diciembre de Universidades. Recuperado de https://www.boe.es/buscar/pdf/2001/BOE-A-2001-24515-consolidado.pdf

López-Justicia, M.D., Hernández, C.M., Fernández, C., Polo, M.T. y Chacón, H. (2008). Características formativas y socioafectivas del alumnado de nuevo ingreso en la Universidad. Revista Electrónica de Investigación Psicoeducativa, 14(6), 95-116.

Mesa, M.C. y Mingorance, A. (2006). Análisis sociológico y expectativas profesionales de los estudiantes de Magisterio de la Facultad de Educación y Humanidades de Melilla. Publicaciones, 36, 36-53. Recuperado de http://digibug.ugr.es/bitstream/10481/28465/1/539.\%20n.\%2036.pdf

Prieto, R. (2001). El perfil del maestro de primaria especialista en educación musical. Revista Interuniversitaria de Formación del Profesorado, 40, 175-185.

Real Decreto 1393/2007, de 29 de octubre, por el que se establece la ordenación de las enseñanzas universitarias oficiales. Recuperado de https://www.boe.es/buscar/pdf/2007/BOE-A-200718770-consolidado.pdf

Real Decreto 1892/2008, de 14 de noviembre, por el que se regulan las condiciones para el acceso a las enseñanzas universitarias oficiales de grado y los procedimientos de admisión a la Universidades Públicas Españolas. Recuperado de https://www.boe.es/buscar/pdf/2008/BOE-A2008-18947-consolidado.pdf

Zurita, F., Padial, R., Viciana, V., Martinez, A., Hinojo, M.A. y Cepero, M. (2016). Perfil del estudiante de Educación Física en el grado de Primaria. Revista Electrónica de Investigación Educativa, 18(2), 156-169. 
